\title{
A case of rheumatoid arthritis that healed completely after repeated intradermal injections with non-specific antigen preparations
}

\author{
Kimihiko Okazaki* \\ Okazaki Medical Clinic, Ukyoku, Kyoto, Japan
}

\section{Introduction}

According to the traditional concept of the contemporary Immunology, neither autoimmune diseases nor allergic diseases can be cured completely. Nevertheless, a fortunate coincidence led the author to discover a novel concept that eliminations of the causes of these diseases are possible. In other words, combinations of pathogenic antibodies with responsible cells, namely, cytolytic $\mathrm{T}$ lymphocytes in cases of autoimmune diseases and mast cells in cases of allergic diseases, can be decomposed by replacing the pathogenic antibodies with non-specific antibodies. In more detail, intradermal injections with a non-specific antigen preparation induce productions of non-specific antibodies in the body of the patient. Repetitions of the injections bring about an accumulation of them. Accumulated nonspecific antibodies will occupy most of the receptors on the surface of responsible cells. When the accumulation reaches the sufficient level, virtually no pathogenic antibodies would remain on the receptors. That is, no causes of the diseases remain.

It is well established that the etiology of allergic diseases is that combinations of mast cells and allergen-specific antibodies cause allergic symptoms when the patients meat allergens. Similarly, the etiology of auto-immune diseases is that combinations of cytolytic $\mathrm{T}$ lymphocytes and organ-specific antibodies cause injury of the organ. A most plain idea would be that break down of the above-mentioned combinations must bring about disappearance of causes of the diseases. To work out the above-mentioned concept, it is necessary to have the patients make non-specific antibodies for themselves. In order for the patients to do so, they need to receive intradermal injections with nonspecific antigen preparations. Consequently, non-specific antibodies accumulate in the patients' bodies, which may replace specific antibodies from respective cells bringing about elimination of causes of the diseases. Needless to mention, where there is no cause, there is no disease. Details are demonstrated elsewhere [1]. The conceptual basis of antibodies' mutual exchange is existence of equilibrium state among antibody molecules in the vicinity of receptors, which was first proposed by Porter [2]. One of the contemporary trends concerning treatments of allergic diseases is an intravenous infusion of solution of non-specific antibody preparation. However, a large number of these infusions are dangerous because anti-antibody antibody might be produced in the recipient's body, which may cause an anaphylactic reaction.

\section{Case report}

A 51-year-old woman (S.Y.) visited the author's clinic on November 2,2016 . She said that she had pains of chin, neck, both-sides shoulders, hand-wrists, fingers, knees, toes, and foot-soles since November 2015. She received an intradermal injection with $0.1 \mathrm{ml}$ of 10 to the 30 -fold with saline diluted Neurotropin; a product of Nippon Zohki Pharmaceutical Company (Osaka), consisting of an extract of rabbit skin inflamed by inoculation of Vaccinia virus, on November 2, 2016 at her navel-edge. On November 4, 2016, she claimed that she had had worse pain at her left shoulder and elbow for 7 hours after the previous injection. She received intradermal injections with $0.1 \mathrm{ml}$ of 10 to the 36-fold with saline diluted Neurotropin on November 4, 7, and 9, 2016. Although her subjective symptom became a little milder after the injections on November 4 and 7, it became a little worse after 10 hours after the injection on November 9 . She received intradermal injections with $0.1 \mathrm{ml}$ of 10 to the 39 -fold with saline diluted Neurotropin on November 11, 14, 16, and 18, 2016. Although her symptom became a little better after each injection except the last one, after which it became neither better nor worse. She received intradermal injections with 0.1 $\mathrm{ml}$ of 10 to the 43 -fold with saline diluted Neurotropin on November 21 and 24, 2016. Although she was free from any pain for 24 hours after the injection on the $21 \mathrm{st}$, she was so for only 12 hours after the injection on the 24,2016 . She received an intradermal injection with $0.1 \mathrm{ml}$ of 10 to the 45-fold with saline diluted Neurotropin on November 25, 2016. She was free from any pain only for 12 hours after the injection on the 25,2016 . On November 28, 2016, she received an intradermal injection with $0.1 \mathrm{ml}$ of 10 to the 47 -fold with saline diluted Neurotropin. She was free from any pain for 24 hours after the injection.

She kept receiving the same injection at 2 3-day intervals until December 7, 2016. Although she was free from any pain for 12 hours after each injection resuming the same level of pain afterwards until December 5th, she claimed somewhat worse pain after 12 hours after the injection on December 7. She received an intradermal injection with $0.1 \mathrm{ml}$ of 10 to the 49 -fold with saline diluted Neurotropin on December 9, 2016. She claimed a worse pain immediately after the last injection. She received an intradermal injection with $0.1 \mathrm{ml}$ of 10 to the 52 -fold with saline diluted Neurotropin on December 12, 14, and 16, 2016. She was free from any pain for 36 hours after the injections on the 12th and the 14th. But, after that on the 16th, she was free from any pain only for 12 hours and she had somewhat worse pain thereafter. She received an intradermal injection with $0.1 \mathrm{ml}$ of 10 to the 58 -fold with saline diluted Neurotropin at her navel-edge on December 19, 2016.

Correspondence to: Kimihiko Okazaki, Okazaki Medical Clinic, Ukyoku, Kyoto, Japan, E-mail: ma13081x@ma1.seikyou.ne.jp

Received: March 12, 2018; Accepted: March 20, 2018; Published: April 05, 2018 
Thereafter, she was free from any pain for 12 hours. In the second morning after the last injection, she began to suffer from much worse pain of almost all joints of arms, fingers, legs, and toes. On December 21,2016 , she received an intradermal injection with $0.1 \mathrm{ml}$ of 10 to the 60 -fold with saline diluted Neurotropin at navel-edge. She claimed that she had purpura and pain at the second joint of her right fifth finger and the second joint of her left third finger for several hours after the last injection. She received an intradermal injection at her naveledge with $0.1 \mathrm{ml}$ of 10 to the 63 -fold with saline diluted Neurotropin on December 22. Her symptom became neither better nor worse immediately after the injection. Namely, she kept feeling the pain until the night of December 25.

She felt no pain thereafter. She received an intradermal injection at navel-edge with $0.1 \mathrm{ml}$ of 10 to the 66 -fold with saline diluted Neurotropin on December 26. She was free from any pain for 36 hours after the last injection. She felt a weak pain thereafter. The rate of multiplication of dilution of Neurotropin with saline was gradually increased to prepare the most effective injection-solution. An apparently relevant explanation of the necessity of the above-mentioned increment of dilution multiplication is as follows: As the number of repetition of the intradermal injection increases, the quantity of accumulated nonspecific antibodies increases. Consequently, quantity of additional accumulation needed for the replacement of pathogenic antibodies with non-specific antibodies decreases. Consequently, again, the patient's ability to produce non-specific antibodies becomes weaker. If nonspecific antigen preparation were injected excessively, the excess nonspecific antigen molecules would attract some of non-specific antibody molecules on the surface of responsible cells by the affinity between antigen and its complementary antibody. The affinity mentioned here exists between non-specific antigens and non-specific antibodies, too. Thus, the multiplication-number of dilution of Neurotropin to prepare the intradermal injection-solution for this patient gradually increased until it reached 10 to the 159 -fold. The length of period, during which she was free from any pain, became longer as she approached the end of treatment. In detail, after October 20, 2017, when she received an intradermal injection at her navel-edge with $0.1 \mathrm{ml}$ of 10 to the 156 fold with saline diluted Neurotropin, she was free from any pain for 80 days. On January 10, 2018, she received an intradermal injection at her navel-edge with $0.1 \mathrm{ml}$ of 10 to the 159 -fold by saline diluted Neurotropin. She was free from any pain for 22 days thereafter. She received an intradermal injection at her navel-edge with $0.1 \mathrm{ml}$ of 1000 fold with saline diluted Picibanil; a product of Chugai Pharmaceutical Company(Tokyo) consisting of $0.02 \mathrm{mg}$ of freeze-dried and with Penicillin-treated Streptococcus biogenes, dissolved in $2 \mathrm{ml}$ of sterilized water on February 2, 2018.

\section{Conclusion}

She is free from any pain ever since.

\section{References}

1. Okazaki K (2009) Therapeutic Significance of Non-Specific Antigens as Anti-Allergic and AntiAutoimmune Agents. Pharmacometrics 76: 105-107.

2. Porter RR (1959) The hydrolysis of rabbit gamma-globulin and antibodies by crystalline papain. Biochem J 73: 119-126. [Crossref]

Copyright: (C2018 Okazaki K. This is an open-access article distributed under the terms of the Creative Commons Attribution License, which permits unrestricted use, distribution, and reproduction in any medium, provided the original author and source are credited. 